9 Yew WW, Chang $\mathrm{KC}$, Chau $\mathrm{CH}$. What is the optimal dosage of linezolid in treatment of complicated multidrug-resistant tuberculosis? Eur Respir J 2009; 34: 1492-1494.

10 Schecter GF, Scott C, True L, et al. Linezolid in the treatment of multidrug-resistant tuberculosis. Clin Infect Dis 2010; 50: 49-55.
11 Migliori GB, Richardson MD, Lange C. Of blind men and elephants: making sense of extensively drug-resistant tuberculosis. Am J Respir Crit Care Med 2008; 178: 1000-1001.

DOI: $10.1183 / 09031936.00199809$

\title{
The online Cough Clinic
}

\section{To the Editors:}

The recent paper by DETTMAR et al. [1] describes an important way in which patients with chronic cough (and their general practitioners) can obtain information online as to the probable diagnosis of their coughs and the preferred treatment.

The only three possible diagnoses are reflux (gastro-oesophageal reflux (GOR)), asthma and rhinitis (post-nasal drip syndrome (PNDS)).

We tested the questionnaire with histories of four patients. They had respectively post-viral, cigarette smoking, habit (Tourette's) and upper respiratory tract infection cough. Each was diagnosed as probable GOR.

The paper raises a number of important questions. First, the paper lists 16 questions pointing to the probable diagnoses, which are identified for each question. However, the current online questionnaire (November 2009) adds a further five questions with no indication of the diagnoses they point to, surely essential information. Presumably this change in the questionnaire was made after the paper was submitted, but it means that the results described in the paper cannot be added to any results from the "new" online questionnaire.

Secondly, how "specific" are the questions? For example, can we be certain, as the questionnaire is, that "clearing your throat" points to GOR and that "excess mucus in the throat" points to PNDS? In the printed questionnaire, if the symptoms are in the throat, four questions point to GOR and two point to PNDS, so there may be a bias towards GOR.

Thirdly, we are told that the questions are not weighted in favour of a particular diagnosis, in that adjustments are made for the different numbers of questions pointing to each diagnosis. But we are also told that there are weighting factors for individual questions, but we are not given these. Within each of the three probable diagnoses, what does this mean? For GOR, does cough during or after eating, point more (or less) than clearing the throat, both of which indicate GOR? For asthma, does wheezing have a greater weighting factor than cough on waking? These examples could be multiplied.

Fourthly, the use of the Likert scale may be justified, but it raises well-known problems. It gives a linear output to a nonlinear input that is different for each question. A mild sensation of heartburn is probably as predictive of GOR as is a strong sensation but this may not be true of throat clearing. In other words, there should be adjustments within the scales as well as between them. Were these adjustments considered and made and, if so, what were they?
Fifthly, figures 2 and 3 in the paper give the proportions of patients reporting each of the 16 symptoms. For asthma diagnosis, answers to the selected three questions show a strong preponderance for those subjects "probably having asthma". For PNDS diagnosis, the answers to the selected five questions show a similar strong preponderance for patients "probably having PNDS". This is to be expected. It follows that all the other patients must probably have GOR. But if you look at the eight GOR-pointing questions, each of them shows roughly equal proportions of patients finally diagnosed with GOR, asthma or PNDS. In other words, the questions for GOR are very non-specific (and lack diagnostic value), unlike those for asthma and PNDS. GOR is the diagnosis after eliminating those who have strong signs of asthma or PNDS; and no other causes of chronic cough are recognised.

Sixthly, the current online version asks 21 questions, each with scores $0-5$ (total 105). If someone answers 1 to one question only (total 1 out of 105) he will be given a diagnosis based on a minimal input. Our "habit cough" patient had a total score of 1 ("very mild hoarse voice"), $<1 \%$ of the possible total and was confidently told that she probably had GOR. We need to be told the probabilities of the probable diagnoses in order to assess the method. Additionally, since only $12.4 \%$ of the patients responded to the follow-up questionnaire, it will never be possible to determine its validity.

Seventhly, it is a pity that the "validation study" included only 30 patients and that it was conducted at the home of the chronic cough questionnaire, with probably much the same questions but asked verbally, rather than independently.

Eighthly, we are told on the website that $78.39 \%$ of those who replied to the follow-up questionnaire found that it had "helped their condition". Since only 944 (9.7\%) out of 9,709 patients replied to the follow-up, we don't know whether the other $90.3 \%$ were "helped". We don't know if the $9.7 \%$ were representative and many chronic coughers get better anyway. There were no control groups.

Finally, we are told that the questionnaire is based on the reports of two ERS Task Forces on Cough. The second Task Force [2] scarcely mentions diagnostic questions and the first [3] gives diagnostic signs and symptoms for different causes of chronic cough (as does any textbook) but nowhere evaluates them. (The authors of this letter were members of both Task Forces.)

These are examples and pressure on space may have prevented including this information and its discussion in the paper but they are crucial for interpretation of the results and could have been presented in the online supplement. 
Nevertheless, the authors are to be congratulated on making patients and their GPs think more about the causes of chronic cough.

\section{J. Widdicombe* and G. Fontana ${ }^{\#}$}

*University of London, London, UK. "University of Florence, Florence, Italy.

Correspondence: J. Widdicombe, 116 Pepys Road, London SW20 8NY, UK. E-mail: JohnWiddicombeJ@aol.com

Statement of Interest: None declared.

\section{REFERENCES}

1 Dettmar PW, Strugala V, Fathi H, et al. The online Cough Clinic: developing guideline-based diagnosis and advice. Eur Respir J 2009; 34: 819-824.

2 Morice AH, Fontana GA, Belvisi MG, et al. ERS guidelines on the assessment of cough. Eur Respir J 2007; 29: 1256-1276.

3 Morice AH, Fontana GA, Sovijarvi AR, et al. The diagnosis and management of chronic cough. Eur Respir J 2004; 24: 481-492.

DOI: $10.1183 / 09031936.00178309$

\section{To the Editors:}

We read with great interest the recent article in the European Respiratory Journal by DETTMAR et al. [1] which discussed an online cough diagnostic clinic. There have been few studies of chronic cough in the population so we are grateful to the authors for providing further data on this topic. We do, however, have several qualms regarding their study.

First, our main concern regards how the differential diagnosis between reflux, asthma and rhinitis was achieved. No clinical characteristic of cough (with the exception of moist cough in children) has been found to be useful in determining diagnostic probability [2-4]. DetTMAR et al. [1] use items from various pathology-specific scales to determine the most probable cause of patients' cough. Most of these scales were originally developed and validated to evaluate disease severity, not to determine a cough's aetiology. The items from reflux symptoms index tools were validated to evaluate voice disorders in laryngopharyngeal reflux, not to ascertain a diagnosis of reflux in a patient presenting with a chronic cough. The questionnaire developed by JUPINER et al. [5] aimed to assess asthma control, not to make a diagnosis of asthma in a patient with a chronic cough. Furthermore, items related to cough timing such as "cough when you get out of bed in the morning", "cough brought on by singing or speaking", "cough after lying down", "cough waking you from sleep" are not correlated with a specific aetiology and may indeed be more related to disease severity than to its aetiology [2].

Secondly, DeTTMAR et al. [1] conducted a validation study of their online cough clinic in 30 patients and found a close association between the web-based cough clinic diagnosis and that of the clinician's full work-up. However, they do not describe the criteria used by the clinician to establish the final diagnosis. Were the same questions used in the clinician's assessment? Furthermore, there is no mention of whether the clinician was blinded to the result of the computerised diagnosis. The close association between the two assessments could be due to lack of blinding in the procedure.

Thirdly, chronic obstructive pulmonary disease (COPD) was not included in the online algorithm. $41.4 \%$ of included patients were either current or ex-smokers. Smokers with shortness of breath and cough could also be suffering from COPD.

Finally, the study concludes that patients with asthma had worse cough scores than those with reflux or rhinitis. This may, however, be a case of secondary association. The diagnosis of asthma in the study was linked to a positive response to the item "cough waking you from sleep", and lack of sleep is the most important cough consequence affecting quality of life.

\section{S. Leconte and J. Degryse}

Dept of General Practice, Université Catholique de Louvain, Brussels, Belgium.

Correspondence: S. Leconte, Dept of General Practice, Université Catholique de Louvain, Avenue Mounier 53, Brussels, 1200, Belgium;. E-mail: sophie.leconte@uclouvain.be

Statement of Interest: None declared.

\section{REFERENCES}

1 Dettmar PW, Strugala V, Fathi H, et al. The online Cough Clinic: developing guideline-based diagnosis and advice. Eur Respir J 2009; 34: 819-824.

2 Mello CJ, Irwin RS, Curley FJ. Predictive values of the character, timing, and complications of chronic cough in diagnosing its cause. Arch Intern Med 1996; 156: 997-1003.

3 Marchant JM, Masters IB, Taylor SM, et al. Utility of signs and symptoms of chronic cough in predicting specific cause in children. Thorax 2006; 61: 694-698.

4 Leconte S, Paulus D, Degryse J. Prolonged cough in children: a summary of the Belgian primary care clinical guideline. Prim Care Respir J 2008; 17: 206-211.

5 Juniper EF, O'Byrne PM, Guyatt GH, et al. Development and validation of a questionnaire to measure asthma control. Eur Respir 1999; 14: 902-907.

DOI: $10.1183 / 09031936.00195509$

\section{From the authors:}

We are grateful to J. Widdicombe and G. Fontana, and S. Leconte and J. Degryse, for providing a detailed critical analysis of our online Cough Clinic programme [1]. However, we believe they have essentially missed the point of the endeavour. We set out to tackle the thorny issue of translating the guidelines into advice accessible to members of the public. Such an enterprise will never provide the precision of a dissected animal, but to dismiss our mainly positive feedback because it is from a mere 944 patients is not only partial but is also a failure to appreciate inherent methodological differences. 